\title{
DEVELOPMENTAL DIFFERENCES IN MYOCYTE CONTRACTILE RESPONSE AFTER CARDIOPLEGIC ARREST
}

William S. McMahon, $\mathrm{MD}^{\mathrm{a}}$

Paul C. Gillette, MD ${ }^{\mathrm{a}}$

Robert B. Hinton, BS ${ }^{\mathrm{b}}$

Jennifer R. Stratton, $\mathrm{BS}^{\mathrm{b}}$

Fred A. Crawford, MD,

Francis G. Spinale, $\mathrm{MD}, \mathrm{PhD}^{\mathrm{b} *}$

\begin{abstract}
Although developmental differences in left ventricular function after cardioplegic arrest and rewarming have been postulated, whether differences exist at the level of the myocyte remains unexplored. This project tested the hypothesis that there is a differential effect of hypothermic hyperkalemic cardioplegic arrest with subsequent rewarming on contractile function of immature compared with adult ventricular myocytes. Myocytes were isolated from the left ventricular free wall of five immature and five adult rabbits and incubated for 2 hours in hyperkalemic modified Ringer's solution at $4^{\circ} \mathrm{C}$ (cardioplegia) or for 2 hours in cell culture medium at $37^{\circ} \mathrm{C}$ (normothermia). Myocytes were resuspended ("rewarmed") in $37^{\circ} \mathrm{C}$ cell culture medium after the incubation protocol. Normothermic baseline contractile performance was lower in immature, compared with adult, myocytes. Specifically, myocyte shortening velocity was $62 \pm 4 \mu \mathrm{m} / \mathrm{sec}$ in immature and $112 \pm 6 \mu \mathrm{m} / \mathrm{sec}$ in adult myocytes $(p<0.01)$. After cardioplegia and rewarming, immature myocyte contractile function was unchanged, whereas adult myocyte contractile function was significantly diminished. For example, myocyte shortening velocity was $65 \pm 4 \mu \mathrm{m} / \mathrm{sec}$ in immature and $58 \pm 3 \mu \mathrm{m} / \mathrm{sec}$ in adult myocytes $(p<0.01$ versus normothermic). Myocyte surface area, which reflects myocyte volume, was increased after cardioplegia and rewarming in adults $(3582 \pm 55$ versus $3316 \pm 46 \mu \mathrm{m}^{2}, p<0.01$ ), but remained unchanged in immature myocytes (2212 \pm 27 versus $2285 \pm 28 \mu \mathrm{m}^{2}, p=$ not significant). These unique findings demonstrate a preservation of myocyte contractile function and volume regulation in immature myocytes after cardioplegic arrest and rewarming. Thus this study directly demonstrates that developmental differences exist in myocyte responses to hypothermic hyperkalemic cardioplegic arrest with subsequent rewarming. (J Thorac Cardiovasc Surg 1996;111:1257-66)
\end{abstract}

eft ventricular (LV) pump dysfunction in the Learly postoperative period is a major source of morbidity and mortality in neonates and infants after palliative and corrective operation for congen-

From the Divisions of Pediatric Cardiology a and Cardiothoracic Surgery, ${ }^{\mathrm{b}}$ Medical University of South Carolina, Charleston, S.C.

Supported in part by National Institutes of Health Training Grant HL07710 (P.C.G.) and HL45024 (F.G.S.).

Received for publication June 22, 1995; accepted for publication August 29, 1995.

Address for reprints: Francis G. Spinale, MD, $\mathrm{PhD}$, Division of Cardiothoracic Surgery, Medical University of South Carolina, 171 Ashley Ave., Charleston, SC 29425.

*Established Investigator of the American Heart Association.

Copyright (C) 1996 by Mosby-Year Book, Inc.

$0022-5223 / 96 \$ 5.00+0 \quad \mathbf{1 2 / 1 / 6 8 9 1 5}$ ital heart disease. ${ }^{1-3}$ One potential contributory mechanism for reduced LV pump function in the early postoperative period is transient changes in myocardial contractile performance as a consequence of cardioplegic arrest and subsequent rewarming.

It has been clearly demonstrated that in the intact, mature LV myocardium, hypothermic hyperkalemic cardioplegic arrest and rewarming causes transient changes in LV pump performance. ${ }^{4-7}$ More recently, it has been demonstrated that hypothermic hyperkalemic cardioplegic arrest and rewarming has direct negative effects on myocyte contractile function in myocytes isolated from mature LV myocardium. ${ }^{8}$ However, there are significant differences between adult and immature myocardium with respect to anatomic and physiologic parameters re- 
lated to energy production and utilization and excitation-contraction coupling. Specifically, immature myocardium has greater potential for anaerobic glycolysis, greater amino acid utilization via substrate-level phosphorylation, and a relatively underdeveloped sarcoplasmic reticulum., 9-13 Despite these developmental differences in myocyte physiologic makeup, fundamentally similar myocardial preservation techniques are used for both adult and immature hearts undergoing cardiac operation. ${ }^{1-3}$ However, whether there is a differential effect of cardioplegic arrest and rewarming on adult and immature myocyte contractile performance has not been explored. Accordingly, the current study was done to test the central hypothesis that differences exist in the response of myocytes isolated from immature hearts compared with the response of myocytes from adult hearts after hypothermic hyperkalemic cardioplegic arrest with subsequent rewarming.

\section{Methods}

LV myocytes were isolated from five adult and five immature rabbits. Isolated myocytes were then randomized to simulated hypothermic hyperkalemic cardioplegic arrest with subsequent rewarming or to incubation under normothermic control conditions. Isolated myocyte contractile function, myocyte response to $\beta$-adrenergic receptor stimulation, and myocyte morphometry were then examined.

Experimental model and myocyte isolation. LV myocytes were isolated from five adult (age 9 months, $4 \mathrm{~kg}$ ) and five sexually immature (age 1 month, $1 \mathrm{~kg}$ ) New Zealand White rabbits. The rabbits were anesthetized (pentobarbital sodium, $50 \mathrm{mg} / \mathrm{kg} ; 2 \%$ isoflurane) and, through a median sternotomy, the heart was expeditiously removed and placed in an iced, oxygenated Krebs solution.

Methods for myocyte isolation after retrograde collagenase digestion have been published previously. ${ }^{8}$ Briefly, the right ventricular free wall and atrial tissue were rapidly dissected from the LV. The aorta was identified and cannulated with the tip of the cannula above the aortic valve. The LV was then perfused with oxygenated modified Krebs solution containing aerobic substrates, hyaluronidase $(1.1 \mathrm{mg} / \mathrm{ml}$, Sigma, St. Louis, Mo.), and collagenase $(1 \mathrm{mg} / \mathrm{ml}$, Worthington Biochemical Corp., Freehold, N.J.) for 20 minutes. The interventricular septum was dissected free and discarded, and the LV free wall myocardium was minced into $2 \mathrm{~mm}^{2}$ sections. These pieces were added to an oxygenated trituration solution containing $400 \mu \mathrm{mol} / \mathrm{L} \mathrm{CaCl}$ and collagenase and gently agitated. In 5-minute intervals, the supernatant was removed and filtered and the cells allowed to settle. The resultant myocyte pellet was then resuspended in cell culture medium (medium M199, $2 \mathrm{mmol} / \mathrm{L} \mathrm{Ca}^{2+}$, Gibco Laboratories, Grand Island, N.Y.). With use of this method, a high yield $(70 \%)$ of viable myocytes was routinely obtained.

All animals were treated and cared for in accordance with the "Guide for the Care and Use of Laboratory Animals" published by the National Institutes of Health (NIH Publication No. 86-23).

Treatment protocols. This laboratory has previously reported a method to subject isolated myocytes to simulated hypothermic hyperkalemic cardioplegic arrest and rewarming. ${ }^{8}$ Briefly, myocytes were randomly assigned to either hypothermic hyperkalemic cardioplegic arrest with subsequent rewarming or to incubation under normothermic control conditions. Myocytes were assigned to either the cardioplegia group, with incubation in modified Ringer's solution $\left(24 \mathrm{mEq} / \mathrm{L} \mathrm{K}^{+}, 30 \mathrm{mEq} / \mathrm{L} \mathrm{HCO}_{3}{ }^{-}, \mathrm{pH} 7.5\right)$ for 2 hours at $4^{\circ} \mathrm{C}$, or to the normothermia group, with incubation in cell culture medium $\left(5.4 \mathrm{mEq} / \mathrm{L} \mathrm{K}^{+}, \mathrm{pH} 7.5\right)$ for 2 hours at $37^{\circ} \mathrm{C}$. The incubation solutions had previously been oxygenated with $95 \% \mathrm{O}_{2}$ and $5 \% \mathrm{CO}_{2}$ to maintain the oxygen tension greater than 300 torr throughout the incubation period. After the 2-hour incubation, rewarming and reperfusion were simulated by resuspension of myocytes in both groups in fresh, oxygenated, normothermic cell culture medium. After rewarming, subsets of myocytes were prepared for contractile function studies or were preserved in a $2 \%$ formaldehyde and $2 \%$ glutaraldehyde solution ( $\mathrm{pH} 7.4,325 \mathrm{mOsm})$ for subsequent morphometric analysis.

Isolated myocyte function. Isolated myocytes were placed in a thermostatically controlled chamber $\left(37^{\circ} \mathrm{C}\right)$ fitted with a coverslip on the bottom for imaging on an inverted microscope (Axiovert IM35, Zeiss Inc., Munich, Germany). Myocytes were imaged with a $\times 20$ long working distance objective. Myocyte contractions were elicited by field stimulation at $1 \mathrm{~Hz}$ (S11, Grass Instruments, Quincy, Mass.) with current pulses of 5 msec duration and voltages $10 \%$ higher than contraction threshold. The polarity of the stimulating electrodes was alternated at every pulse to prevent the buildup of electrochemical by-products. Myocyte contractions were imaged with use of a charge-coupled device with a noninterlaced scan rate of $240 \mathrm{~Hz}$ (model GPCD60, Panasonic, Secaucus, N.J.). Myocyte motion signals were captured with a single myocyte aligned parallel to the video raster lines, and this video signal was input through an edge-detector system (Crescent Electronics, Sandy, Utah). The distance between the left and right myocyte edges was converted into a voltage signal, digitized, and input to a computer (80486, Zeos International, Minneapolis, Minn.) for subsequent analysis.

Stimulated myocytes were allowed a 5-minute stabilization period after which contraction data for each myocyte were recorded from a minimum of 20 consecutive contractions. Parameters computed from the digitized contraction profiles included percent shortening, peak velocity of shortening (in micrometers per second), peak velocity of relengthening (micrometers per second), total contraction duration (milliseconds), time to peak contraction (milliseconds), and time to $50 \%$ relaxation (milliseconds). After collection of these baseline indices of myocyte function, measurements were then repeated after the 

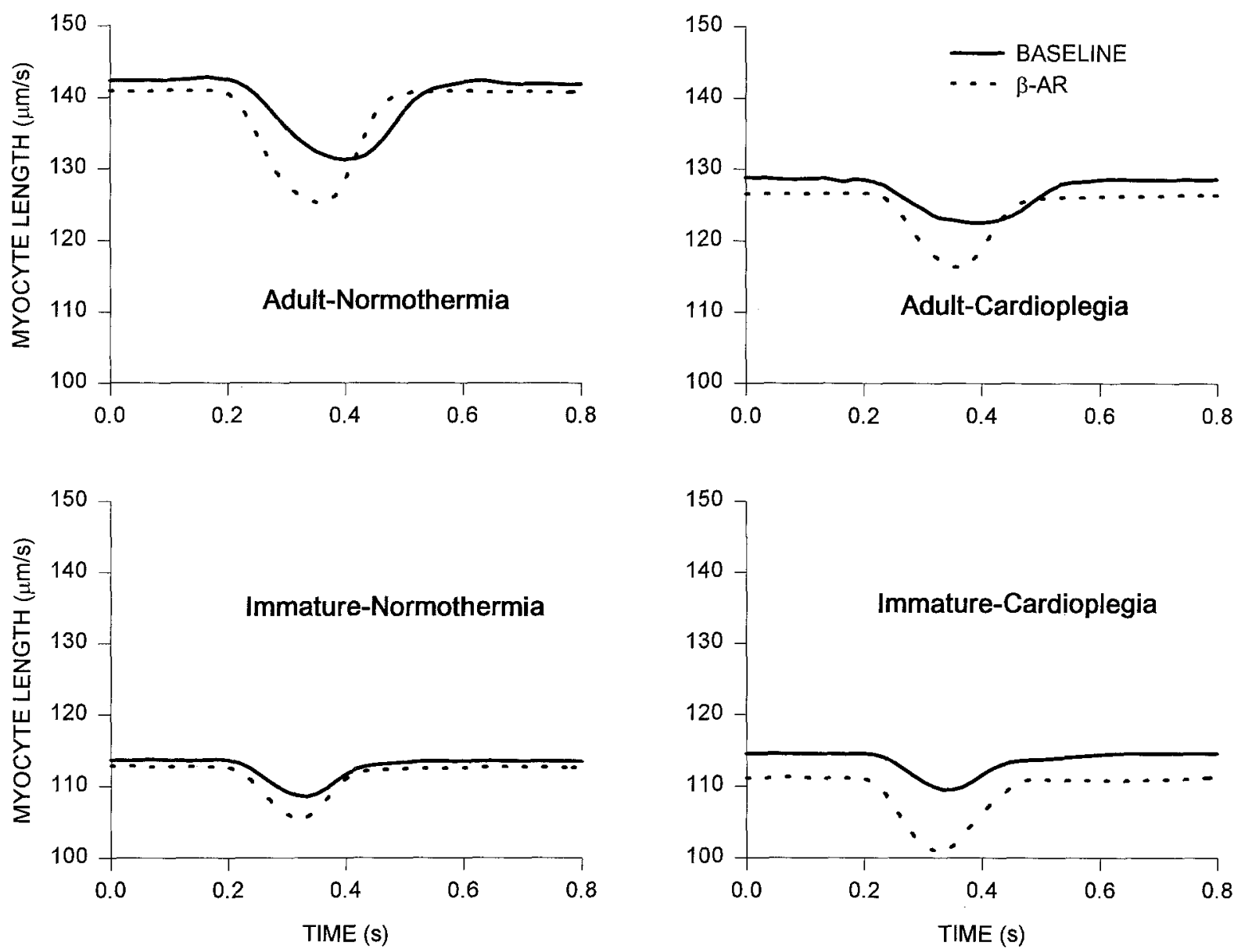

Fig. 1. Representative myocyte contraction profiles at baseline and after $\beta$-adrenergic receptor stimulation $(\beta-A R)$ from adult and immature myocytes after normothermic incubation or hypothermic hyperkalemic cardioplegic arrest with rewarming. In adult myocytes, resting length and contractile performance were decreased after cardioplegic arrest and rewarming. In contrast, immature myocyte resting length and contractile performance were unchanged after cardioplegic arrest and rewarming. Indices of myocyte contractile function are summarized in Tables I and II.

addition of (-)-isoproterenol $(25 \mathrm{nmol} / \mathrm{L})$ to the chamber. ${ }^{14}$

Isolated myocyte morphometry. To measure myocyte surface area, previously preserved myocytes were examined under light microscopy on an inverted microscope (model IM-35, Zeiss). Myocytes were imaged at $\times 1000$ magnification with use of an epifluorescence illuminator with a rhodamine filter. Isolated myocyte borders were digitized by an image analysis system (IBAS, Zeiss/Kontron), and profile surface area was computed by planimetry directly from the digitized image. It has been previously demonstrated that the profile surface area directly reflects myocyte volume. ${ }^{15}$

Data analysis. Indices of myocyte function were compared among the treatment groups by analysis of variance. Analysis of the morphometric data was done with the average measurements obtained for each animal, and the groups were compared by analysis of variance. If the analysis of variance revealed significant differences, pairwise tests of individual group means were compared by Bonferroni probabilities. ${ }^{16}$ All statistical procedures were done with a BMDP statistical software package (BMDP Statistical Software Inc., Los Angeles, Calif.). Data are presented as mean plus or minus the standard error of the mean. Values of $p<0.05$ were considered to be statistically significant.

\section{Results}

Isolated myocyte contractile function. Representative baseline contraction profiles for adult and immature myocytes after incubation under normothermic conditions and after hypothermic hyperkalemic cardioplegic arrest with subsequent rewarming are demonstrated in Fig. 1. A summary of 
Table I. Effects of hypothermic hyperkalemic cardioplegic arrest and rewarming in adult and immature rabbit myocytes

\begin{tabular}{lcc}
\hline & Normothermia & Cardioplegia \\
\hline Resting length $(\mu \mathrm{m})$ & $140 \pm 2$ & $126 \pm 2^{*}$ \\
$\quad$ Adult & $114 \pm 2 \dagger$ & $114 \pm 2 \dagger$ \\
$\quad$ Immature & & \\
Percent shortening $(\%)$ & $7.3 \pm 0.2$ & $4.4 \pm 0.2^{*}$ \\
$\quad$ Adult & $4.4 \pm 0.3 \dagger$ & $5.3 \pm 0.2^{*} \dagger$ \\
$\quad$ Immature & $112 \pm 6$ & $58 \pm 3^{*}$ \\
Shortening velocity $(\mu \mathrm{m} / \mathrm{sec})$ & $62 \pm 4 \dagger$ & $65 \pm 4$ \\
$\quad$ Adult & & \\
Immature & $125 \pm 6$ & $59 \pm 3^{*}$ \\
Relengthening velocity $(\mu \mathrm{m} / \mathrm{sec})$ & $63 \pm 4 \dagger$ & $75 \pm 5 \dagger$ \\
$\quad$ Adult & & \\
Immature & $394 \pm 9$ & $386 \pm 9$ \\
Duration of contraction $(\mathrm{msec})$ & $308 \pm 12 \dagger$ & $370 \pm 21^{*}$ \\
$\quad$ Adult & & \\
Immature & $202 \pm 5$ & $189 \pm 3^{*}$ \\
Time to peak contraction $(\mathrm{msec})$ & $150 \pm 5 \dagger$ & $176 \pm 4^{*} \dagger$ \\
$\quad$ Adult & & \\
Immature & & $73 \pm 3$ \\
Time to $50 \%$ relaxation $(\mathrm{msec})$ & $57 \pm 3 \dagger$ & $64 \pm 3 \dagger$ \\
$\quad$ Adult \\
Immature
\end{tabular}

Normothermia, 2-hour incubation (cell medium, $5.4 \mathrm{mEq} / \mathrm{L} \mathrm{K}^{+}$) at $37^{\circ} \mathrm{C}$; Cardioplegia, 2-hour incubation (crystalloid, $24 \mathrm{mEq} / \mathrm{L} \mathrm{K}^{+}$) at $4^{\circ} \mathrm{C}$; Adult, New Zealand White rabbit, age 6 months, $4 \mathrm{~kg}$; Immature, age 1 month, $1 \mathrm{~kg}$.

* $p<0.05$ versus Normothermia

$\dagger p<0.05$ versus Adult.

isolated myocyte contractile function at baseline after normothermic incubation and after hypothermic hyperkalemic cardioplegic arrest and rewarming is shown in Table I. Under normothermic baseline conditions, immature myocyte contractile performance was different from that in adult myocytes. Specifically, shortening velocity was $45 \%$ lower in immature myocytes when compared with the adult myocyte values. After hypothermic hyperkalemic cardioplegic arrest with subsequent rewarming, adult myocyte contractile function was significantly diminished compared with the normothermia values. For example, adult myocyte shortening velocity was $48 \%$ lower after cardioplegic arrest and rewarming. In contrast, immature myocyte shortening velocity was not diminished after hypothermic hyperkalemic cardioplegic arrest and rewarming compared with the normothermia value. Interestingly, immature myocyte percent shortening was higher after hypothermic hyperkalemic cardioplegic arrest with subsequent rewarming compared with normo- thermia control values. In both adult and immature myocytes, there was no change in the number of viable myocytes after cardioplegic arrest when compared with normothermia values.

Myocyte peak relengthening velocity, an index of active relaxation processes ${ }^{17}$ was also derived from the contraction profiles. Under normothermic baseline conditions, immature myocyte relengthening velocity was lower, compared with adult myocyte values. After hypothermic hyperkalemic cardioplegic arrest and rewarming, adult myocyte relengthening velocity was diminished compared with the normothermia control values. In contrast, immature myocyte relengthening velocity was unchanged after hypothermic hyperkalemic cardioplegic arrest with subsequent rewarming. Another important index of myocyte contractile function, the total duration of contraction, was shorter in immature myocytes after normothermic incubation than in adult myocytes. After hypothermic hyperkalemic cardioplegic arrest with subsequent rewarming, duration of contraction was unchanged in adult myocytes, but was increased in immature myocytes. Thus the increased percent shortening observed in immature myocytes after hypothermic hyperkalemic cardioplegic arrest with subsequent rewarming was a function of preserved shortening and relengthening velocities with increased total duration of contraction.

Myocyte $\boldsymbol{\beta}$-adrenergic responsiveness. In the next series of studies, the capacity of isolated myocytes to respond to an inotropic stimulus was examined after exposure to the $\beta$-adrenergic agonist isoproterenol. Representative contraction profiles for adult and immature myocytes with $\beta$-adrenergic receptor stimulation after incubation under normothermic conditions and after hypothermic hyperkalemic cardioplegic arrest with subsequent rewarming are shown in Fig. 1. Indices of isolated myocyte contractile function with $\beta$-adrenergic stimulation after normothermic incubation and after hypothermic hyperkalemic cardioplegic arrest and rewarming are shown in Table II. In the presence of isoproterenol, isolated myocyte contractile function was increased, compared with baseline values, in adult and immature myocytes after normothermic incubation and after hypothermic hyperkalemic cardioplegic arrest and rewarming. After incubation under normothermic conditions, the $\beta$-adrenergic response was lower in immature myocytes than in adult myocytes. Specifically, shortening velocity was $37 \%$ lower in immature myocytes compared with that in adult myocytes. After hypothermic hy- 
perkalemic cardioplegic arrest and rewarming, adult myocyte $\beta$-adrenergic response was diminished compared with normothermic control values. For example, shortening velocity was $44 \%$ lower in adult myocytes after hypothermic hyperkalemic cardioplegic arrest and rewarming. In contrast, immature myocyte $\beta$-adrenergic response after hypothermic hyperkalemic cardioplegic arrest with subsequent rewarming was not diminished compared with normothermia control values.

Isolated myocyte geometry. Adult and immature isolated myocyte resting lengths are summarized in Table I. After incubation under normothermic conditions, isolated myocyte resting length was greater in adult, compared with immature, myocytes. After hypothermic hyperkalemic cardioplegic arrest with subsequent rewarming, resting length in adult myocytes was significantly reduced. In contrast, immature myocyte resting length was unchanged after hypothermic hyperkalemic cardioplegic arrest and rewarming.

To further investigate changes in myocyte geometry after hypothermic hypokalemic cardioplegic arrest and rewarming, myocyte profile surface area was measured. Myocyte surface area frequency distributions for adult and immature myocytes after normothermic incubation and after hypothermic hyperkalemic cardioplegic arrest and rewarming are shown in Fig. 2. The frequency distribution approximated a gaussian distribution in all groups. Normothermic adult myocyte profile surface area (3316 \pm $46 \mu \mathrm{m}^{2}$ ) was significantly higher than that for immature myocytes $\left(2212 \pm 27 \mu \mathrm{m}^{2}, p<0.01\right)$. After hypothermic hyperkalemic cardioplegic arrest with subsequent rewarming, adult myocyte surface area was increased $\left(3582 \pm 55 \mu \mathrm{m}^{2}, p<0.01\right)$. In contrast, there was no change in immature myocyte profile surface area after hypothermic hyperkalemic cardioplegic arrest and rewarming $\left(2285 \pm 28 \mu \mathrm{m}^{2}\right.$, $p<0.01$ ). Thus in adult myocytes hypothermic hyperkalemic cardioplegic arrest and rewarming caused decreased resting length, but significantly increased myocyte surface area. These changes in myocyte geometry are consistent with myocyte swelling. ${ }^{15}$ In contrast, no significant changes in immature myocyte resting length or surface area occurred.

\section{Discussion}

LV pump dysfunction occurs in all age groups after major cardiac operations. Though usually transient, the resulting low cardiac output state may
Table II. Hypothermic hyperkalemic cardioplegic arrest and rewarming in adult and immature rabbit myocytes: effect of $\beta$-adrenergic receptor stimulation

\begin{tabular}{|c|c|c|}
\hline & Normothermia & Cardioplegia \\
\hline \multicolumn{3}{|c|}{ Percent shortening (\%) } \\
\hline Adult & $9.9 \pm 0.5^{*}$ & $6.7 \pm 0.3^{* \dagger}$ \\
\hline Immature & $6.2 \pm 0.4^{*} \ddagger$ & $8.1 \pm 0.3^{*}+\ddagger$ \\
\hline \multicolumn{3}{|c|}{ Shortening velocity $(\mu \mathrm{m} / \mathrm{sec})$} \\
\hline Adult & $188 \pm 16^{*}$ & $105 \pm 6^{*} \dagger$ \\
\hline Immature & $93 \pm 7^{*} \neq$ & $110 \pm 5^{*} \dagger$ \\
\hline \multicolumn{3}{|c|}{ Relengthening velocity $(\mu \mathrm{m} / \mathrm{sec})$} \\
\hline Adult & $202 \pm 14^{*}$ & $106 \pm 6^{*} \dagger$ \\
\hline Immature & $98 \pm 7^{*} \ddagger$ & $124 \pm 7^{*}+\frac{1}{*}$ \\
\hline \multicolumn{3}{|c|}{ Duration of contraction (msec) } \\
\hline Adult & $309 \pm 11^{*}$ & $340 \pm 11^{*}$ \\
\hline Immature & $275 \pm 8^{*}+4$ & $311 \pm 14^{*} \dagger$ \\
\hline \multicolumn{3}{|c|}{ Time to peak contraction (msec) } \\
\hline Adult & $160 \pm 5^{*}$ & $165 \pm 3^{*}$ \\
\hline Immature & $143 \pm 4+$ & $155 \pm 4^{*} \dagger$ \\
\hline \multicolumn{3}{|c|}{ Time to $50 \%$ relaxation $(\mathrm{msec})$} \\
\hline Adult & $54 \pm 2^{*}$ & $61 \pm 2^{*} \dagger$ \\
\hline Immature & $51 \pm 2$ & $54 \pm 2^{*}+$ \\
\hline \multicolumn{3}{|c|}{ Sample size ( $n=$ myocytes $)$} \\
\hline Adult & 105 & 129 \\
\hline Immature & 44 & 49 \\
\hline
\end{tabular}

Nomothermia, 2-hour incubation (cell medium, $5.4 \mathrm{mEq} / \mathrm{L} \mathrm{K}^{+}$) at $37^{\circ} \mathrm{C}$; Cardioplegia, 2-hour incubation (crystalloid, $24 \mathrm{mEq} / \mathrm{L} \mathrm{K}^{+}$) at $4^{\circ} \mathrm{C} ;$ Adult, New Zealand White rabbit, age 6 months, $4 \mathrm{~kg}$; Immature, age 1 month, $1 \mathrm{~kg}$.

${ }^{*} p<0.05$ versus baseline

$\uparrow p<0.05$ versus Normothermia

$\neq p<0.05$ versus Adult.

result in significant morbidity and mortality. ${ }^{1-3}$ In adult myocardium, hypothermic hyperkalemic cardioplegic arrest with subsequent rewarming has been linked to transient postoperative LV pump dysfunction..$^{4-7}$ This laboratory has previously demonstrated that diminished LV myocyte contractile performance after exposure to hypothermic hyperkalemic cardioplegia with subsequent rewarming is a contributory mechanism for diminished $L V$ function. ${ }^{8}$ In immature myocardium, however, there is experimental evidence for increased resistance to the detrimental effects of ischemia. ${ }^{18-22}$ In addition, developmental differences exist between adult and immature myocardium that may account for increased ischemic tolerance of the immature heart. ${ }^{9-13}$ However, the direct effects of hypothermic hyperkalemic cardioplegic arrest with subsequent rewarming on immature myocyte contractile performance remained unknown.

Accordingly, the current study addressed this question with use of a method to subject isolated myocytes to simulated cardioplegic arrest and rewarming. There are several unique findings of this 

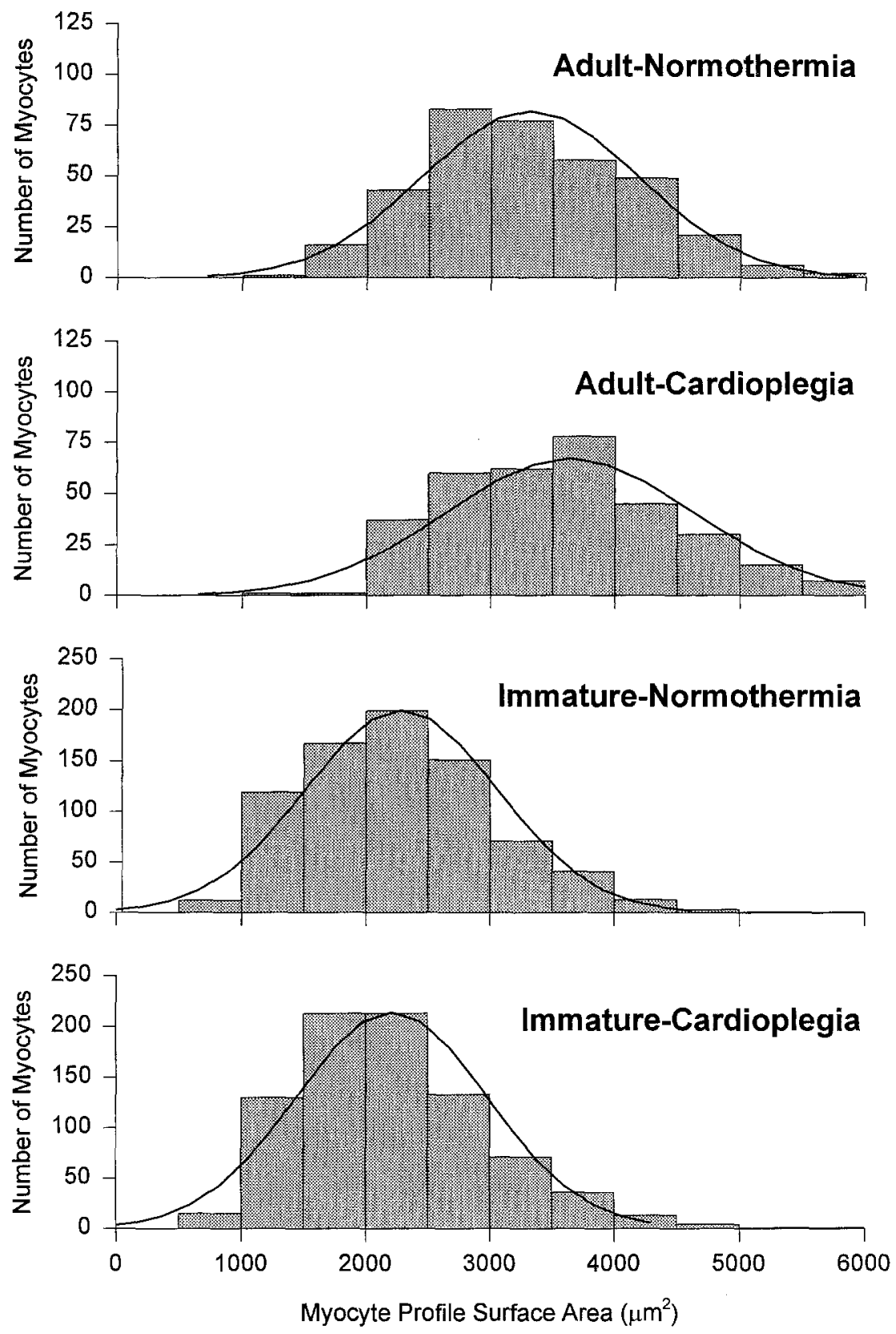

Fig. 2. Myocyte profile surface area frequency distribution for adult and immature myocytes after normothermic incubation or hypothermic hyperkalemic cardioplegic arrest and rewarming. Myocyte profile surface area directly reflects myocyte volume. Normothermic baseline profile surface area was greater in adult than in immature myocytes. After hypothermic hyperkalemic cardioplegic arrest and rewarming, adult myocyte profile surface area was significantly increased. In contrast, there was no change in immature myocyte surface area after cardioplegic arrest and rewarming. Absolute values for mean myocyte profile surface area are given in Results section.

study. First, unlike that of adult ventricular myocytes, immature myocyte contractile function was not diminished by hypothermic hyperkalemic cardioplegic arrest with subsequent rewarming. Sec- ond, unlike that of adult myocytes, contractile response to $\beta$-adrenergic receptor stimulation was not diminished in immature myocytes after hypothermic hyperkalemic cardioplegic arrest and rewarming. 
Third, immature myocyte volume was not affected by exposure to cardioplegia and rewarming, which implies preservation of cellular homeostasis. Thus this study provides, for the first time, direct evidence that there is an age-dependent, differential response of myocyte contractile function after hypothermic hyperkalemic cardioplegic arrest and rewarming.

The clinical and basic literature that addresses ischemia and cardioplegic protection in the immature myocardium is controversial. For example, several clinical studies have reported more favorable results in immature, compared with adult, myocardium after hypothermic cardioplegia, ${ }^{23-25}$ whereas others have reported inadequate protection in immature myocardium. ${ }^{26-28}$ Experimental studies have also produced conflicting results. ${ }^{9-11,18-22}$ In both clinical and experimental studies, a wide range of subject ages, models, cardioplegic protocols, and endpoint measures may have contributed to the disparate findings. For instance, in experimental studies of immature hearts, time to ischemic contracture and capacity for functional recovery as different endpoint measures may produce conflicting results in the same model. ${ }^{13}$ In the current study, these complexities are simplified by study of the basic measure of contractile function, the myocyte. To our knowledge, this study is the first to examine the age-dependent effects of hypothermic cardioplegia and rewarming on myocyte contractile function. This study provides direct evidence that immature myocytes are more resistant, compared with adult myocytes, to the detrimental effects on contractile performance of hypothermic hyperkalemic cardioplegic arrest with subsequent rewarming.

Isolated myocyte contractile function. In the current study, under normothermic baseline conditions, immature myocyte contractile performance was significantly lower than that in adult myocytes. This result is consistent with previous reports of lower developed tension and lower maximal rate of tension development in immature, compared with adult, myocardium. ${ }^{29}$ Also consistent with these findings, immature myocytes have been demonstrated to have lower myofibril content, reduced myofibrillar adenosinetriphosphatase levels, and lower intracellular calcium concentrations compared with adult myocytes. ${ }^{30}$ After hypothermic hyperkalemic cardioplegic arrest with subsequent rewarming, contractile function was reduced in adult myocytes. This finding is consistent with that of a previous report from this laboratory. ${ }^{8}$ The unique finding of this study is the demonstration that contractile function in immature myocytes was not diminished after hypothermic hyperkalemic cardioplegic arrest and rewarming. This study provides new evidence to suggest that a fundamental mechanism of greater functional recovery of immature myocardium after hypothermic hyperkalemic cardioplegic arrest and rewarming is preservation of myocyte contractile function.

Hyperkalemic cardioplegic arrest results in increased intracellular calcium concentrations. ${ }^{31}$ Previous studies have reported that the resulting intracellular calcium overload is a major contributing factor to the development of postischemic myocardial injury. ${ }^{32,33}$ Because of age-related underdevelopment of the sarcoplasmic reticulum, it has been proposed that reduced ability to sequester increased intracellular calcium with hyperkalemic cardioplegic arrest may potentiate myocardial injury in immature hearts. ${ }^{2}$ However, because there are developmental differences in cellular calcium handling, differences in the calcium response to increased extracellular potassium may exist. In fact, it has been reported that potassium-induced depolarization of immature myocytes does not result in significantly increased intracellular calcium levels. ${ }^{34}$ Thus a potential contributory mechanism for the developmental difference in myocyte contractile response after hypothermic hyperkalemic cardioplegic arrest and subsequent rewarming is age-dependent differences in myocyte calcium physiologic makeup and reduced calcium-mediated myocyte injury.

Myocyte $\boldsymbol{\beta}$-adrenergic responsiveness. In the current study, $\beta$-adrenergic receptor stimulation of adult myocytes after incubation under normothermic control conditions caused increased contractile performance. Immature myocyte contractile function also increased after $\beta$-adrenergic receptor stimulation, but remained lower than that in adult myocytes. As previously stated with respect to differences between immature and adult baseline contractile function, this finding is consistent with known functional immaturity of cellular contractile components such as the sarcoplasmic reticulum and myofilaments. Consistent with a previous report, hypothermic hyperkalemic cardioplegic arrest with subsequent rewarming caused reduced adult myocyte $\beta$-adrenergic responsiveness. ${ }^{8}$ In contrast, there was not a reduction in immature myocyte $\beta$-adrenergic responsiveness after hypothermic hyperkalemic cardioplegic arrest and rewarming. It is likely that the mechanism for this preservation of $\beta$-adrenergic responsiveness in immature myocytes is the 
same as that discussed for increased baseline contractile function, namely reduced calcium overload in immature myocytes with potassium-induced cardioplegia. However, another possible mechanism for this age-dependent effect of $\beta$-adrenergic receptor stimulation after cardioplegia and rewarming is abnormalities of intracellular transduction. It has been demonstrated that one mechanism for increased contractility after $\beta$-adrenergic receptor stimulation is generation of cyclic adenosine monophosphate and subsequent phosphorylation of the sarcoplasmic reticulum-bound protein phospholamban. ${ }^{35}$ In addition, reductions in sarcoplasmic reticulum content and its adenosinetriphosphatase activity are characteristic of immature myocytes. ${ }^{29}$ Taken together, these past reports and the current study suggest that hypothermic hyperkalemic cardioplegic arrest with subsequent rewarming may have a selective effect on sarcoplasmic reticulum activity and cyclic adenosine monophosphate-dependent processes that would have less impact on immature myocyte $\beta$-adrenergic responsiveness.

Isolated myocyte geometry. The current study measured myocyte profile surface area, which is proportional to myocyte volume, ${ }^{15}$ in adult and immature myocytes after hypothermic hyperkalemic cardioplegic arrest with subsequent rewarming. This analysis demonstrated increased myocyte volume in adult, but not immature, myocytes after cardioplegic arrest and rewarming. These findings suggest preservation of myocyte volume regulation in immature myocytes after exposure to hypothermic hyperkalemic cardioplegic arrest and rewarming. The mechanisms responsible for volume regulation in the cell are adenosine triphosphate dependent. ${ }^{15}$ Immature myocytes have been proposed to have greater capacity for energy production via glycosylation and amino acid phosphorylation pathways. ${ }^{9-11,30}$ Thus one potential mechanism for maintenance of myocyte volume regulation in immature myocytes in the current study is enhanced adenosine triphosphate production and utilization during prolonged hyperkalemic arrest. Although the mechanism responsible for volume regulation during hypothermic hyperkalemic cardioplegic arrest and rewarming remains speculative, the maintenance of myocyte volume was associated with preservation of myocyte performance.

Limitations. There are several limitations to the current study that must be recognized. As with any model of myocardial protection, this model does not exactly duplicate the clinical physiologic conditions of aortic crossclamping and administration of hypothermic cardioplegic solution. However, the method does provide a unique means to study the direct effects of cardioplegia on myocardial contractility at the level of the myocyte. The isolated myocyte system for the in vitro study of contractile function offers several unique advantages. Specifically, in vivo hemodynamic and neurohormonal influences, which may also be specifically altered in the postbypass period, are eliminated as possible confounding factors. In addition, this model is independent of potential problems with cardioplegic solution distribution caused by coronary stenoses. In the current study, significant differences in myocyte contractile function were observed between immature and adult cardiocytes after cardioplegic arrest. However, fundamental mechanisms that contributed to these differences remain speculative and warrant further investigation. Specifically, whether differential effects in the function of the sarcolemmal and sarcoplasmic reticulum occur between adult and immature myocytes after cardioplegic arrest remains to be established. In this study, myocyte contractile function was examined in unloaded cardiocytes taken from mature and immature ventricles. Thus the capacity of these myocytes to respond to an external workload was not examined. However, in a past report it was demonstrated that changes in unloaded myocyte velocity of shortening is linearly related to changes in the capacity of these myocytes to contract against an external load. ${ }^{36}$ Therefore the diminished myocyte velocity of shortening that occurred in the adult myocytes after cardioplegic arrest in the current study suggests intrinsic defects in the capacity of these cardiocytes to respond to an external load.

Direct comparison of animal to human ages and developmental stages is problematic. However, the age of the animals used in this study falls well within a previously published "immature" category. ${ }^{30}$ In the human context, differences exist between this model and the usual clinical situations in which cardioplegia is used for adults and young infants. Specifically, cardioplegia for the immature heart is typically used in the context of significant preexisting cardiac pathologic conditions such as heart failure, myocardial hypertrophy from volume or pressure overload, or chronic cyanosis. Nevertheless, the focus of the current study was to test the central hypothesis that there are developmental differences in myocyte responses to hypothermic hyperkalemic cardioplegic arrest with subsequent rewarming. 
Summary. This study provides unique evidence to suggest that there is a developmental difference in the myocyte contractile response to hypothermic hyperkalemic cardioplegic arrest with subsequent rewarming. Specifically, after simulated cardioplegic arrest and rewarming, adult myocyte contractile function and cellular homeostasis were significantly impaired. In contrast, after cardioplegic arrest and rewarming, immature myocyte contractile function, $\beta$-adrenergic responsiveness, and cellular homeostatic mechanisms for volume regulation were preserved.

\section{REFERENCES}

1. Kirklin JK, Kirklin JW, Pacifico AD. Intraoperative myocardial protection. In: Arciniegas E, ed. Pediatric cardiac surgery. Chicago: Year Book Medical, 1985:87-94.

2. Diaco M, DiSesa VJ, Sun SC, Laurence R, Cohn LH. Cardioplegia for the immature myocardium. J Thorac Cardiovase Surg 1990;100:910-3.

3. Buckberg GD. Myocardial protection: an overview. Semin Thorac Cardiovasc Surg 1993;5:98-106.

4. Roberts AJ, Spies SM, Sanders JH, et al. Serial assessment of left ventricular performance following coronary artery bypass grafting. J Thorac Cardiovasc Surg 1981;81:69-84.

5. Phillips HR, Carter JE, Okada RD, et al. Serial changes in left ventricular ejection fraction in the early hours after aortocoronary bypass grafting. Chest 1983;83:28-34.

6. Mjos OD, Ichihara K, Fellenius E, Myrmel T, Neely JR. Fatty acids suppress recovery of heart function after hypothermic perfusion. Ann Thorac Surg 1991;52:965-70.

7. Mankad PS, Severs NJ, Lachno DR, Rothery S, Yacoub MH. Superior qualities of University of Wisconsin solution for in vivo preservation of the pig heart. J Thorac Cardiovasc Surg 1992;104:229-40.

8. Handy JR, Spinale FG, Mukherjee R, Crawford FA. Hypothermic potassium cardioplegia impairs myocyte recovery of contractility and inotropy. J Thorac Cardiovasc Surg 1994; 107:1050-8.

9. Julia PL, Kofsky ER, Buckberg GD, Young HH, Bugyi HI. Studies of myocardial protection in the immature heart: I-enhanced tolerance of immature versus adult myocardium to global ischemia with reference to metabolic differences. J Thorac Cardiovasc Surg 1990;100:879-87.

10. Julia PL, Young HH, Buckberg GD, Kofsky ER, Bugyi HI. Studies of myocardial protection in the immature heart: II-evidence for importance of amino acid metabolism in tolerance to ischemia. J Thorac Cardiovasc Surg 1990;100: 888-95.

11. Breuer E, Barta E, Pappova E, Zlatos L. Developmental changes of myocardial metabolism: I-peculiarities of cardiac carbohydrate metabolism in the early postnatal period in dogs. Biol Neonate 1967;11:367-77.

12. Boland R, Martonosi A, Tillack TW. Developmental changes in the composition and function of the sarcoplasmic reticulum. J Biol Chem 1974;249:612-23.

13. Quantz M, Tchervenkov C, Chiu RC. Unique responses of immature hearts to ischemia. J Thorac Cardiovase Surg 1992;103:927-35.
14. Spinale FG, Tempel GE, Mukherjee R, et al. Cellular and molecular changes in the $\beta$-adrenergic receptor system with supraventricular tachycardia induced cardiomyopathy. Cardiovasc Res 1994;28:1243-50.

15. Drewnowska K, Baumgarten CM. Regulation of cellular volume in rabbit ventricular myocytes: bumetanide, chlorothiazide, and ouabain. Am J Physiol 1991;260:C122-31.

16. Steel RGD, Torrie JH. Principles and procedures of statistics: a biometrical approach. 2nd ed. New York: McGrawHill, 1980:1-623.

17. Zile MR, Mukherjee R, Clayton C, Kato S, Spinale FG. Effects of chronic supraventricular pacing tachycardia on relaxation rate in isolated cardiac muscle cells. Am J Physiol 1995;268:H2104-13.

18. Baker JE, Boerboom LE, Olinger GN. Age-related changes in the ability of hypothermia and cardioplegia to protect ischemic rabbit myocardium. J Thorac Cardiovasc Surg 1988; 96:717-24.

19. Coles JG, Watanabe T, Wilson GJ, et al. Age-related differences in the response to myocardial ischemic stress. J Thorac Cardiovase Surg 1987;94:526-34.

20. Ganzel BL, Katzmark SL, Mavroudis C. Myocardial preservation in the neonate: beneficial effects of cardioplegia and systemic hypothermia on piglets undergoing cardiopulmonary bypass and myocardial ischemia. J Thorac Cardiovasc Surg 1988;96:414-22.

21. Konishi T, Apstein CS. Comparison of three cardioplegic solutions during hypothermic-ischemic arrest in neonatal blood-perfused rabbit hearts. J Thorac Cardiovasc Surg 1989;98:1132-7.

22. Magovern JA, Pae WE, Waldhausen JA. Protection of the immature myocardium: an experimental evaluation of topical cooling, single-dose, and multiple-dose administration of St. Thomas' Hospital cardioplegic solution. J Thorac Cardiovasc Surg 1988;96:408-13.

23. Kirklin JK, Blackstone EH, Kirklin JW, McKay R, Pacifico $\mathrm{AD}$, Bargeron LM. Intracardiac surgery in infants under age 3 months: incremental risk factors for hospital mortality. Am J Cardiol 1981;48:500-6.

24. Crawford FA, Barnes TY, Heath BJ. Potassium-induced cardioplegia in patients undergoing correction of congenital heart defects. Chest 1980;78:316-20.

25. Burrows FA, Williams WG, Teoh $\mathrm{KH}$, et al. Myocardial performance after repair of congenital cardiac defects in infants and children: response to volume loading. $\mathbf{J}$ Thorac Cardiovasc Surg 1988;96:548-56.

26. Bull C, Cooper J, Stark J. Cardioplegic protection of the child's heart. J Thorac Cardiovasc Surg 1984;88:287-93.

27. Del Nido PJ, Mickle DAG, Wilson GJ, et al. Inadequate myocardial protection with cold cardioplegic arrest during repair of tetralogy of Fallot. J Thorac Cardiovasc Surg 1988;95:223-9.

28. Sawa Y, Matsuda H, Shimazake Y, et al. Ultrastructural assessment of the infant myocardium receiving crystalloid cardioplegia. Circulation 1987;76(Suppl):V141-5.

29. Nakanishi T, Jarmakani JM. Developmental changes in myocardial mechanical function and subcellular organelles. Am J Physiol 1984;246:H615-25.

30. Riva E, Hearse DJ. The developing myocardium. New York: Futura, 1991.

31. Powell T, Tatham PER, Twist VW. Cytoplasmic free calcium measured by quin2 fluorescence in isolated ventricular myo- 


\section{McMahon et al.}

The Journal of Thoracic and Cardiovascular Surgery June 1996 cytes at rest and during potassium-depolarization. Biochem Biophys Res Commun 1984;122:1012-20.

32. Cheung JY, Bonventre JV, Malis CD, Leaf A. Calcium and ischemic injury, N Engl J Med 1986;314:1670-6.

33. Murphy JG, Marsh JD, Smith TW. The role of calcium in ischemic myocardial injury. Circulation 1987;75(Suppl):V1524.

34. Cyran SE, Phillips J, Ditty S, Baylen BG, Cheung J, LaNoue $K$. Developmental differences in cardiac myocyte calcium homeostasis after steady-state potassium depolarization: mechanisms and implications for cardioplegia. J Pediatr 1993;122:S77-83.

35. Feher JJ, Fabiato A. Cardiac sarcoplasmic reticulum: calcium uptake and release. In: Langer GA, ed. Calcium and the heart. New York: Raven Press, 1990.

36. Urabe Y, Mann DL, Kent RL, et al. Cellular and ventricular contractile dysfunction in experimental canine mitral regurgitation. Circ Res 1992;70:131-47. 